\title{
The Attribute Accuracy Assessment of Land Cover Data in the National Geographic Conditions Survey
}

\author{
Xiaole $\mathrm{Ji}^{\text {a, }}$, Xiao Niu ${ }^{\text {a }}$ \\ Shandong Provincial Institute of Land Surveying and Mapping \\ Jinan, Shandong Province, China \\ (jixiaole218, niuxiao710)@gmail.com
}

KEY WORDS: Land cover, Accuracy assessment, National geographic survey, Error matrix, Object-based classification, Weight, High-resolution remotely sensed imagery

\begin{abstract}
:
With the widespread national survey of geographic conditions, object-based data has already became the most common data organization pattern in the area of land cover research. Assessing the accuracy of object-based land cover data is related to lots of processes of data production, such like the efficiency of inside production and the quality of final land cover data. Therefore, there are a great deal of requirements of accuracy assessment of object-based classification map. Traditional approaches for accuracy assessment in surveying and mapping are not aimed at land cover data. It is necessary to employ the accuracy assessment in imagery classification. However traditional pixel-based accuracy assessing methods are inadequate for the requirements. The measures we improved are based on error matrix and using objects as sample units, because the pixel sample units are not suitable for assessing the accuracy of object-based classification result. Compared to pixel samples, we realize that the uniformity of object samples has changed. In order to make the indexes generating from error matrix reliable, we using the areas of object samples as the weight to establish the error matrix of object-based image classification map. We compare the result of two error matrixes setting up by the number of object samples and the sum of area of object samples. The error matrix using the sum of area of object sample is proved to be an intuitive, useful technique for reflecting the actual accuracy of object-based imagery classification result.
\end{abstract}

\section{INTRODUCTION}

Land is one of the most important resources in economy development. Reasonable uses of land resources affect the mode of society and economy development. Using remote sensing imagery, especially high resolution remote sensing imagery, to access to the urban land use information, becomes indispensable technology. High resolution remote sensing data (e.g., QuickBird, IKONOS, Geo-Eyes and aerial photography) can provide higher definition than those from preceding sensors (e.g., Landsat Thematic Mapper and SPOT). Using these high resolution remote sensing data can obtain higher levels of detailed features of land use and land cover. On the one hand, more detailed features may be conducive to classification (Hay, 2005). On the other hand, finer resolution image data may cause some degrees of redundancy (Blaschke, 2001) and reduce the identification accuracy in urban image classification (Myint, 2006). Despite all that, high resolution remote sensing is still a crucial research direction. One of the most important reasons is the rise of Objectbased classification. Many researchers believe that Object-based classification not only can overcome the shortcoming of high resolution image data, but also can make a better use of the profusion of details to improve the accuracy of classification (Blaschke 2010, Yan, 2006). With widely use of the high resolution imagery, Object-based classification has received an unprecedented development since 2000s (Blaschke 2010).

A national survey of geographic conditions has widespread carried out since 2013. The data origin of this survey is finer than 2.5 meter high-resolution remotely sensed imagery. Land cover map is one type data which has been produced in the project. There are lots of differences between land cover and traditional surveying data, such like DLG, DEM. For example, land cover is organized with a great deal of objects, which have different attribute. Besides, land cover refers to every corner on the ground. This situation brings out a great deal of requirements of accuracy assessment of object-based classification map. In the recently promulgated stipulation of the national geographic condition survey, attribute accuracy of land cover data has the greatest weight. Traditional pixel-based accuracy assessment method has many disadvantages in deal with object-based data. Because that Object-based classification of remote sensing image combines the pixels with similar characteristic into a polygon or object according to a regulation. At the end of classification, the whole image is in expression of objects in different categories.

Error matrix is the classical method in the field of attribute accuracy assessment of remotely sensed imagery. which is also called confusion matrix (Congalton, 1991, Congalton, 2008), has been the major method of attribute accuracy assessment since 1980s'. The process of this method is selecting sample from classification result and comparing the sample with reference data. The samples selected, for a long term, are pixels, which have still been argued since object-based classification developed (Grenier, 2008, Kozak, 2006, Persello, 2006, Qiu, 2010, Zhan 2005). The sample units of object-based classification result are pixels (Qiu, 2010) and polygons (Grenier, 2008, Kozak, 2006). A researcher assesses the accuracy of object-based classification map with these two the sample units and thinks the object sample units can reflect more accuracy information about the objectbased classification map (Zhan, 2005). Congalton and Green also point out that if the map being assessed is an object map, then the accuracy assessment sample units should also be polygons (Congalton, 2008). 
There are three reasons for choosing objects as the sample unit. First of all, object-based classification results are object maps, object samples are much easier to be selected. Secondly, the objects in object-based classification map represent the surface feature, it is very important to judge the entire accuracy of object which can't be realized with pixel units. Finally, object units can get the reference from interpreting results, which can reduce the cost of sampling. When adequate number of object samples per category is gathered, the assessment is a statistically valid representation of the accuracy of the map (Grenier, 2008). In this study, we basically use this method to determine the number of sample polygons but change a little according to the situation.

However, one of the most important differences between object sample and pixel sample ignored by lots of researchers is the changes of consistency of sample units. The pixels in a remote sensing image have the same area, as the result, samples are undifferentiated. In contrast, the area of polygon samples differ from one another, which will affect the result of error matrix.

In this research, we use polygons as sample units and error matrix to assess the attribute accuracy, and consider the sampling number. Besides, we employ the area of each object sample as weight. At the same time, we set up an error matrix without area of weight as comparison.

\section{METHOD}

Because of the shortages of the accuracy assessments at present discussed above, we propose an accuracy assessment method that considers attribute accuracies of object. Based on traditional method of accuracy assessment, we propose the concept of the area weight accuracy, and we use this concept to evaluate the accuracy of object-based classification result. This method that use object as sample can reduce the overestimate or underestimate of the accuracy.

In this section, we simply introduce the object-based at first. Then we discuss two important concepts - error matrix and area weighted, which are the basis of our method. At last, we put forward the basic framework and indexes of accuracy assessment of object-based classification result.

\subsection{Object-based Classification}

Segmentation and classification are two steps to create objects in classification result (Hay, 2008). The segmentation gives an object a region of space (Carleer, 2005) and the classification gives the object the attribute (Castilla, 2008). The relationship between pixel and object is reflected in the process of segmentation and classification. The location and spectrum information implied in pixel is the basis for the segment algorithm. The pixels with same features are assembled by segment algorithm into an object which have spatial and shape information (Baatz, 2008). Finally, the objects are classified according to features of the pixels in segments by classification algorithm, like fuzzy classification method, to give every segment an attribute (Benz, 2004).

Attribute accuracy of object is the root in pixel-based classification accuracy assessment but more complex. Because pixels in traditional pixel-based classification result don't have relation with each other, there is only attribute accuracy which indicates the accordance between pixels in classification map and reference data at the same location (Congalton, 2008). In the object-based classification, attribute accuracy is no longer limited in pixels. Object only has one attribute category within many pixels which may have different attributes (Zhan, 2005, Persello, 2010). So the attribute accuracy assessment of object is more complicated than that of pixels. The attribute accuracy of object is involved with the relationship among reference data, classification object and pixels in object.

There are mainly three differences between attribute accuracy assessment of object-based and pixel-based classification result. Firstly, object in classification result is comprised of pixels, which determine the attribute category of object. This phenomenon may lead to the situation that the uniqueness of object's attributes and the diversity of pixels' attributes exists at the same time, which means the pixel can't represent the object. So accuracy assessment of object-based classification result can't choose pixels as samples. Secondly, if we choose objects as assessing samples, the attribute diversity inside the object still can't be reflected. Although the hypotheses in segmentation is that pixels in a segment have the same attribute, in fact, this assumption is often violated due to the "different objects same image" phenomenon in remote sensing imagery. The last but not least, the classification accuracy of imagery is representing the area percentage of correct classification, which is estimated by the number of sample pixels classified correctly in pixel-based classification result. In object-based classification, the accuracy is reflected by object samples. However, one of most important differences among object samples is their area diversity. According to the characteristic of object-based classification, the imagery with more otherness may get smaller objects in classification, and vice versa. This peculiarity of object-based classification may cause different representativeness among object-samples.

\subsection{Error Matrix}

Error matrix, which is also called confusion matrix, use the classified and reference data to compare and set up a matrix. The indexes, such as the overall accuracy, producer's accuracy and user's accuracy, can be generated from the matrix. (Figure 1).

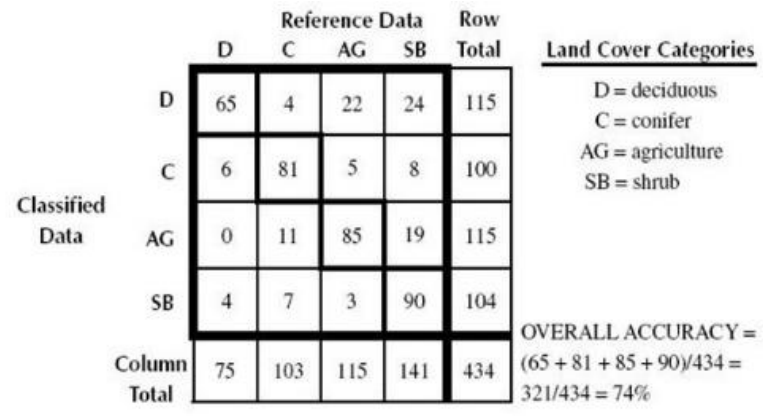

$$
\begin{aligned}
& \text { PRODUCER'S ACCURACY } \\
& \text { D }=65 / 75=87 \% \\
& \mathrm{C}=81 / 103=79 \% \\
& \mathrm{AG}=85 / 115=74 \% \\
& \mathrm{SB}=90 / 141=64 \%
\end{aligned}
$$

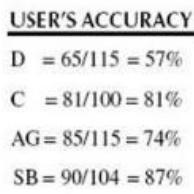

Figure 1 Error Matrix

Error matrix is a very effective way to calculate map accuracy. Overall accuracy is the sum of the major diagonal (the number of correctly classified sample units) divided by the total number of sample units. Producer's and user's accuracy are computed for individual category accuracy by dividing the total number of 
sample units in one category by the total number of sample units in this column or row. A commission error represents an area in a category which it does not belong to that category. An omission error reveals the excluded area from the category to which it belongs to. Producer's accuracy plus omission error equals 1 and user's accuracy plus commission error equals 1 (Congalton, 2008).

The method we proposed still use confusion matrix as the basic form. The reason is that confusion matrix set up according to category can calculate the accuracy of each class and reflect the degree of confusion between classes directly. Besides, the confusion matrix is a concise and straightforward method. No matter how many samples are selected, as long as the number of category is still the same, the number of rows is not changing and the indexes are comparable, which suits land cover data in national geographic condition survey.

\subsection{Sample Size}

Sample size is the adequate number of sample per class gathered, which is determined by the desired precision of the sample representation and the cost of sampling. Congalton and Green consider the sample size is resolved by two principles: category number and the proportion of each category. They recommend the minimum of sample size of each category is 50 sample units (Congalton, 2008).

\subsection{Area Weight}

The purpose of accuracy assessment of classification result of land cover map is to obtain the percentage of correctly classified area, which can also be described by the percentage of total number of pixels labeled to the right attribute. In the accuracy assessment of object-based classification map, because of different scale and regulation, the area of objects is disparity. In general, classified objects have larger area in the homogeneous region and smaller area in heterogeneous region. Pixel sample units have the same sample area, hence the samples in error matrix are undifferentiated. However, this condition can't be found if the sample units become objects. It is unreasonable if the same weight distribute for large object and small one. For this reason, areas of sample units should be adopted as the weight of accuracy assessment.

Considering the producer's and user's accuracy' computation, sample units in each column and row need to be comparable. In this research, we adopt actual area of object sample as the weight to calculate the error matrix.

\section{CASE STUDY}

\subsection{Segmentation and Classification}

Considering security, the imagery we used is a small piece of QuickBird imagery of Dongying, Shandong Province (Figure 2). The spatial resolution is approximately 0.61 meters. The classified data, getting from eCognition and the virtual interpretation (Figure 3), represents the land cover data which needs to assess. And the classification system has been kept the first class only. In the test region, the area of different types varies greatly. The process of sampling is conducted in ArcGIS.

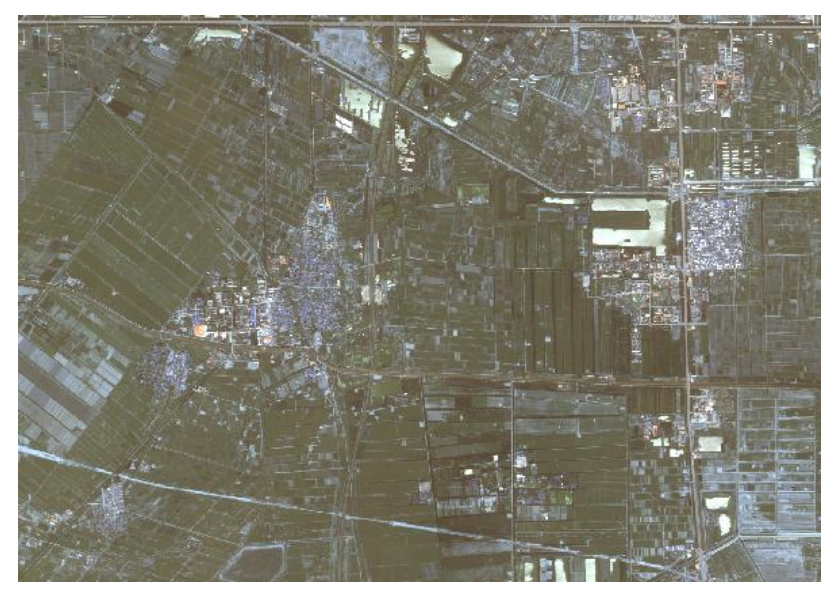

Figure 2 RGB Imagery

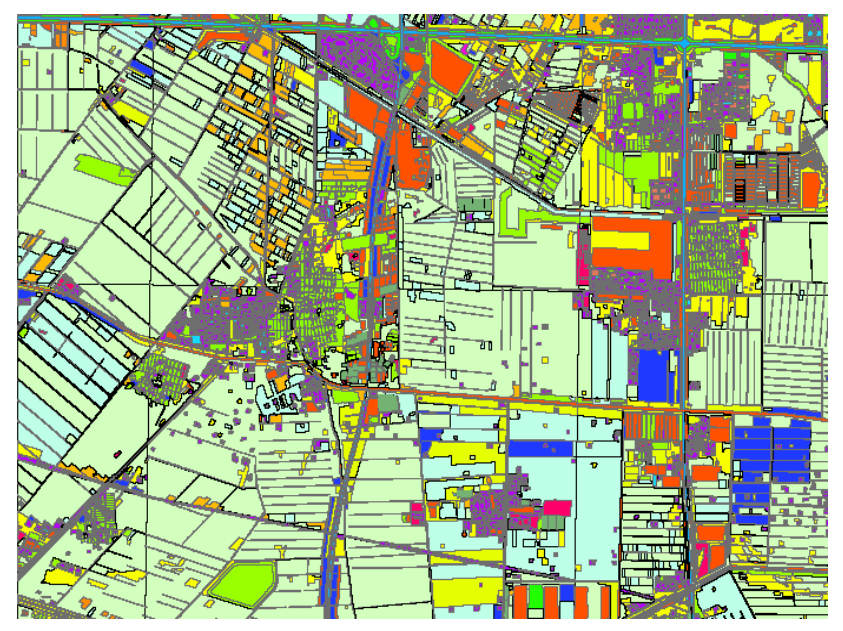

Figure 3 Classification Map

In this study, the stratified random sampling was used. We analyze the object-based classification result and census the maximum area, minimum area, area and percentage area in each category (Table 1). From Table 1, we can get the conclusion that the maximum area is much larger than minimum area in each category. In the national geographic conditions survey, the number from 0100 to 1000 represent plough, garden plot, woodland, grassland, building, road, structures, accumulation, bare land, water area. In the testing area, there is few bare land.

\begin{tabular}{|c|c|c|c|c|c|c|}
\hline Class & $\begin{array}{l}\text { Total area } \\
\left(\mathbf{m}^{2}\right)\end{array}$ & $\begin{array}{l}\% \\
\text { Area }\end{array}$ & $\begin{array}{l}\text { Sample } \\
\text { num. }\end{array}$ & $\begin{array}{l}\text { Sample } \\
\text { area } \\
\left(\mathbf{m}^{2}\right)\end{array}$ & $\begin{array}{l}\text { Maximum } \\
\text { area } \\
\left(\mathbf{m}^{2}\right)\end{array}$ & $\begin{array}{l}\text { Minimum } \\
\text { area } \\
\left(\mathbf{m}^{2}\right)\end{array}$ \\
\hline 0100 & 29918430 & 2.76 & 71 & 824541 & 188356 & 376 \\
\hline 0200 & 5998031 & 2.14 & 43 & 128070 & 22467 & 412 \\
\hline 0300 & 3421536 & 9.71 & 55 & 332079 & 40483 & 451 \\
\hline 0400 & 2456218 & 16.69 & 47 & 409955 & 74582 & 411 \\
\hline 0500 & 4321672 & 12.41 & 41 & 536238 & 2337 & 368 \\
\hline 0600 & 3945490 & 12.88 & 60 & 508287 & 65850 & 413 \\
\hline 0700 & 4417642 & 5.01 & 31 & 221365 & 5038 & 501 \\
\hline 0800 & 3514367 & 18.70 & 52 & 657221 & 32262 & 423 \\
\hline 1000 & 1754309 & 18.41 & 56 & 322902 & 13361 & 389 \\
\hline TOTAL & 59747695 & 6.60 & 456 & 3940658 & 444736 & 3744 \\
\hline
\end{tabular}

Table 1. Distribution of classes based on the area, number of objects, maximum and minimum area 


\subsection{Reference Data}

The reference data used in this study is the field surveying result based on the inner data production, which regard as ground truth. If this method conduct into accuracy assessment of this program, then the reference data will be the field examining which will be executed by quality inspection departments. However, there is an inevitable issue in taking object as sample, which is the same region of object in imagery may contain different types of features. In this research, we regard the largest area of feature in this object as the category of this reference data.

We use random sample to obtain object samples of each class. Every object in a class has the same opportunity to be chosen, no matter the larger object or smaller one. Traditional sampling is based on reference data to determine the number of sample units. In order to ensure that every class has sufficient samples, we choose samples in reference data firstly, and then extract all classified objects which have interaction with the reference data.

\subsection{Error Matrix}

We make use of objects as sample units to establish error matrix. In order to compare difference between the number of object error matrix and the area weighted ones, we set up two error matrixes using the same sample units. Table 2 is the error matrix which cell is the number of object samples. Table 3is the error matrix which cell is the sum of area of object samples. All the accuracy indexes are calculated based on Table 2 and Table 3, including overall accuracy, producer's accuracy and user's accuracy of each category (Table 4).

Reference Data

\begin{tabular}{|l|l|lllllllll|l|}
\hline Class & 0100 & 0200 & 0300 & 0400 & 0500 & 0600 & 0700 & 0800 & 1000 & Total \\
\hline 0100 & 64 & 2 & 1 & 1 & 0 & 2 & 1 & 0 & 0 & 71 \\
0200 & 2 & 35 & 2 & 1 & 1 & 0 & 0 & 1 & 1 & 43 \\
0300 & 2 & 2 & 45 & 2 & 0 & 0 & 2 & 1 & 1 & 55 \\
Data & 0400 & 1 & 2 & 3 & 37 & 2 & 2 & 0 & 0 & 0 & 47 \\
0500 & 0 & 0 & 0 & 2 & 35 & 2 & 1 & 1 & 0 & 41 \\
0600 & 1 & 1 & 2 & 1 & 1 & 47 & 2 & 2 & 3 & 60 \\
0700 & 0 & 0 & 0 & 2 & 4 & 2 & 21 & 2 & 0 & 31 \\
0800 & 1 & 1 & 2 & 1 & 2 & 2 & 3 & 38 & 2 & 52 \\
1000 & 1 & 0 & 1 & 2 & 2 & 1 & 3 & 2 & 44 & 56 \\
\hline Total & 72 & 43 & 56 & 49 & 47 & 58 & 33 & 47 & 51 & 456 \\
\hline
\end{tabular}

Table 2. Error matrix of number of object samples

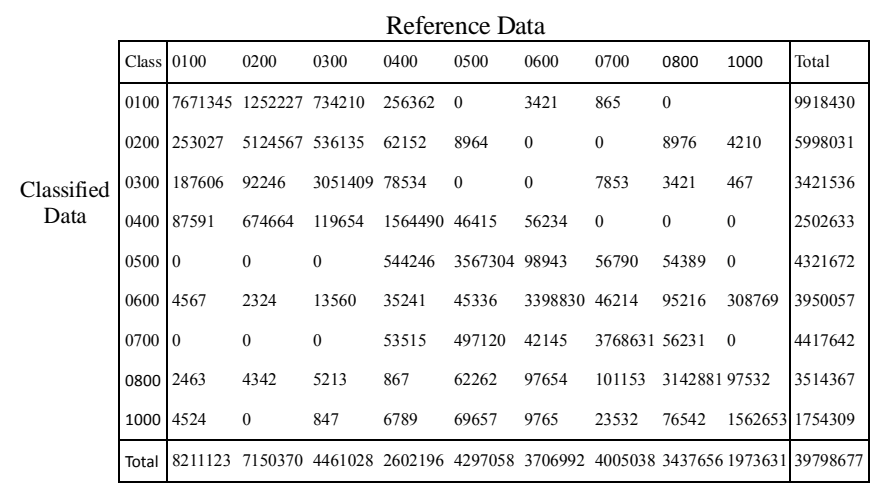

Table 3. Error matrix of sum of area of object samples

\begin{tabular}{|c|c|c|c|}
\hline Classes & Measures & $\begin{array}{l}\text { Error Matrix in } \\
\text { Table } 2\end{array}$ & $\begin{array}{l}\text { Error Matrix in } \\
\text { Table } 3\end{array}$ \\
\hline \multirow[t]{2}{*}{0100} & user's accuracy & $90.14 \%$ & $77.34 \%$ \\
\hline & producer's accuracy & $88.89 \%$ & $93.43 \%$ \\
\hline \multirow[t]{2}{*}{0200} & user's accuracy & $81.40 \%$ & $85.44 \%$ \\
\hline & producer's accuracy & $81.40 \%$ & $71.67 \%$ \\
\hline \multirow[t]{2}{*}{0300} & user's accuracy & $81.82 \%$ & $89.19 \%$ \\
\hline & producer's accuracy & $80.36 \%$ & $68.40 \%$ \\
\hline \multirow[t]{2}{*}{0400} & user's accuracy & $78.72 \%$ & $62.51 \%$ \\
\hline & producer's accuracy & $75.51 \%$ & $60.12 \%$ \\
\hline \multirow[t]{2}{*}{0500} & user's accuracy & $85.37 \%$ & $82.54 \%$ \\
\hline & producer's accuracy & $74.47 \%$ & $83.02 \%$ \\
\hline \multirow[t]{2}{*}{0600} & user's accuracy & $78.33 \%$ & $86.05 \%$ \\
\hline & producer's accuracy & $81.03 \%$ & $91.69 \%$ \\
\hline \multirow[t]{2}{*}{0700} & user's accuracy & $67.74 \%$ & $85.31 \%$ \\
\hline & producer's accuracy & $63.64 \%$ & $94.10 \%$ \\
\hline \multirow[t]{2}{*}{0800} & user's accuracy & $73.08 \%$ & $89.43 \%$ \\
\hline & producer's accuracy & $80.85 \%$ & $91.43 \%$ \\
\hline \multirow[t]{2}{*}{1000} & user's accuracy & $80.26 \%$ & $89.08 \%$ \\
\hline & producer's accuracy & $86.27 \%$ & $79.18 \%$ \\
\hline 0800 & Overall accuracy & $80.26 \%$ & $82.55 \%$ \\
\hline
\end{tabular}

Table 4. Accuracy indexes based on table 2 and table 3

We could get from Table 2, Table 3 and Table 4 that different forms of error matrix will lead to quite different results of accuracy indexes. For example, the overall accuracy of area object error matrix is $2.29 \%$ higher than the number object error matrix. And a greater difference can be seen from each category's producer's accuracy and user's accuracy. The category of 0700 , the difference between Table 2 and Table 3 up to more than $20 \%$. The reverse situation happens on the category of 0400 . The user's accuracy in Table 2 is $16.21 \%$ higher than that in Table 3 . The producer's accuracy of 0300 in Table 2 is $11.96 \%$ higher than that in Table 3, and the user's accuracy of 0100 in Table 2 is $12.8 \%$ higher than that in Table 3. These results reflect that large objects are classifying incorrectly. There is one object in reference data of 0300 with area up to $734210 \mathrm{~m}^{2}$ is classified incorrectly into 0100 in classified data, and two objects with area up to 536135 $\mathrm{m}^{2}$ is classified into 0200 in classified data. The same situation happens in category of 0100 . A few of big objects are classifying incorrectly bring about those categories assessing results.

Besides, comparing to Table 2, the user's and producer's accuracy indexes of the same category in Table 3 vary widely. The difference between user's and producer's accuracy is up to $20.79 \%$ in category of 0300 . The value difference shows that objects of 0300 in classified data can be recognized well, but there are lots of objects which do not belong to 0300 going for this category. Considering the size of misallocated objects, the indexes in Table 2 failed to reflect the situation. The larger difference between user's and producer's accuracy indexes, the greater size of objects exist in uncorrected classified categories. We can see the categories of 0100, 0200, 0700, 1000 have the same case. 
The reason of situation above is the origin of objects. Big objects in classified map are generated from adjacent objects with the same category. Before the progress of merging, those objects can be seen as different objects. For further consideration, the objects without merging are put into assessment, then the result of Table 2 will be close to Table 3 . In Table 3 , the error matrix is setting up based on the number of objects, and the incorrect small objects have large influence on the assessing result. If we take the extreme case for example, $1 \mathrm{~m}^{2}$ object can be classified in a remotely sensed imagery, which is put into a wrong category. At the same time, a $100000 \mathrm{~m}^{2}$ object is also mis-classified. In Table 2 , the $1 \mathrm{~m}^{2}$ object has the same weight as $100000 \mathrm{~m}^{2}$ object, which is obviously unreasonable. We can get the conclusion that the indexes of number error matrix may underestimate or overestimate the actual accuracy of object oriented imagery classification result and the accuracy indexes of area weight error matrix is more reliable.

Another advantage of this method is the direct presentation of different mistaken classifying. There always have some categories which are differentiated easily from the other categories. For example, it is easily distinguished water area from road, and it is not very easy to differentiate road and bare land. For this reason, it is a more serious mistake of confusing road and water than confusing road and bare land. In the error matrix, the categories confusing pattern is shown in a straightforward way.

\section{CONCLUSIONS}

There is not a perfect method to assess the accuracy of objectbased classification result now. Every method has both advantages and disadvantages. Comparing to other methods, this method neither overestimate nor underestimate the accuracy of object-based classification.

An area-weighted error matrix was suggested for the attribute accuracy assessment of the land cover map is reviewed. Error matrix is the widely used method in the field of remotely sensed image classification assessing. With the object-based classification becoming the most popular method, the error matrix needs improve.

The object sample units were selected to establish error matrix. Because of the area difference of every object sample units, there are two ways to make use of the sample information. The first one is to count the number of correctly classified objects in each category, which is treating object sample units the same as pixel units. The second method is to consider that the great area difference of object samples in each category. In this research, we compare these two methods of establishing error matrix. It is proved that adopting the number of object sample units will bring about overestimating or underestimating the accuracy of objectbased imagery classification map and indexes getting from area weighting error matrix is more reliable.

As a result, we use area of object samples as the weight to establish the error matrix. We make use the sum of area of object samples instead of the number of objects samples classified correctly or wrongly to setting up error matrix. However, it is necessary to admit that the object sample unit can bring about uncertainties because the object sample is regarded as uniformity in the region, which is just a hypothesis and not working all the time. This problem is worth of further study.

\section{REFERENCES}

Blaschke, T. (2010) Object based image analysis for remote sensing. ISPRS Journal of Photogrammetry and Remote Sensing, 65, 2-16.

Blaschke, T. \& J. Strobl (2001) What's wrong with pixels? Some recent developments interfacing remote sensing and GIS. GIS Zeitschrift für Geoinformationssysteme 14(6), 12-17.

Yan, G., M. J. F, B. H. P. Maathuis \& X. Zhang (2006) Comparison of Pixel-based and Object-oriented image Classification Approaches-A Case Study in a Coal Fire Area, Wuda, Inner Mongolia,China International Journal of Remote Sensing, 17-27.

Congalton G., "A review of assessing the accuracy of classifications of remotely sensed Data." Remote Sensing of Environment, 1991.37(1), pp. 35-46.

Congalton G., Green K., "Assessing the Accuracy of Remotely Sensed Data: Principles and Practices." [M]. 2008. CRC Press Taylor \& Francis Group

Grenier M., Labrecque S., Benoit M., Allard M., "Accuracy assessment method for wetland object-based classification." In: Proceedings GEOBIA, 2008-Pixels, Objects, Intelligence: GEOgraphic Object Based Image Analysis for the 21st Century. pp. 285289Kozak J.,

Estreguil C., Vogt P., "Forest cover and pattern changes in the Carpathians over the last decades." European Journal of Forest Research. 2006. Vol. 126 77-90

Kozak J., Estreguil C., Vogt P. "Forest cover and pattern changes in the Carpathians over the last decades." European Journal of Forest Research. 2006.Vol. 126 77-90

Persello C., Bruzzone L., "A Novel Protocol for Accuracy Assessment in Classification of Very High Resolution Images." IEEE Transactions on Geoscience and Remote Sensing. 2010.Vol. 48, 1232-1244

Qiu J., Wang X.. “A Comparative Study on Object-based Land Cover Classification in High Spatial Resolution Remote Sensing Imagery of Urban Areas." Remote Sensing Technology and Application 2010, Vol. 25 No.5

Zhan Q., Molenaar M., Tempfli K., and Shi W., "Quality assessment for geo-spatial objects derived from remotely sensed data." International Journal of Remote Sensing, 2005. 26(14), 2953-2974

Hay, G. J. \& G. Castilla. 2008. Geographic Object-Based Image Analysis (GEOBIA): A new name for a new discipline. In Object-Based Image Analysis, ed. S. L. a. G. J. H. T. Blaschke, 75-89.

Carleer, A. P., O. Debeir \& E. Wolff (2005) Assessment of Very High Spatial Resolution Satellite Image Segmentations. Photogrammetric Engineering \& Remote Sensing 71, 1285-1294.

Castilla, G. \& G. J. Hay. 2008. Image objects and geographic objects. In Object-Based Image Analysis, ed. S. L. a. G. J. H. T. Blaschke, 91-110.

Baatz, M., C. Hoffmann \& G. Willhauck. 2008a. Progressing from object-based to object-oriented image analysis. In Objectbased Image Analysis, ed. T. Blaschke.

Benz, U. C., P. Hofmann, G. Willhauck, I. Lingenfelder \& M. Heynen (2004) multi-resolution, object-oriented fuzzy analysis 
ISPRS Annals of the Photogrammetry, Remote Sensing and Spatial Information Sciences, Volume II-4, 2014 ISPRS Technical Commission IV Symposium, 14 - 16 May 2014, Suzhou, China

of remote sensing data for GIS-ready information. ISPRS Journal of Photogrammetry \& Remote Sensing, 58, 19.

\section{ACKNOWLEDGMENT}

This research is supported by the National Geographic Condition Survey in Shandong Province. 